\title{
INFLUENCIA DE LA PROMOCIÓN DE EXPORTACIONES EN EL DESARROLLO EXPORTADOR DE LAS MYPES DE LA REGIÓN TACNA, PERIODO 2008 - 2009
}

\author{
Autora: Renzi Marilu Loza Ticona ${ }^{1}$ \\ Universidad Privada de Tacna \\ E-mail: renpis@hotmail.com
}

\section{RESUMEN}

La investigación es de tipo básica, de naturaleza descriptiva correlacional debido a que busca determinar la relación de las variables de estudio: Promoción de exportaciones y Desarrollo Exportador. Para tal propósito se consideró la información obtenida a través de la aplicación del cuestionario "Promoción de Exportaciones y Desarrollo Exportador de las MYPES de la Región Tacna”, el cual fue aplicado a 99 Mypes exportadoras de la Región Tacna.

El objetivo fue determinar la influencia de la promoción de exportaciones en el desarrollo exportador de las MYPES de la Región Tacna, periodo 2008-2009.

Los resultados más importantes son: las MYPES exportadoras de la Región Tacna tienen un bajo conocimiento sobre los beneficios que brindan los organismos de promoción de exportaciones; las MYPES exportadoras de la Región Tacna acceden a los programas y servicios de la promoción de exportaciones con menor frecuencia anualmente; los objetivos de alianza con los organismos de promoción de exportaciones de las MYPES de la Región Tacna, fueron en mayor medida hacia la información y capacitación sobre exportaciones; $y$, asesoría y exportaciones.

Asimismo, el nivel de los programas y servicios brindado por los organismos de promoción de exportaciones es bajo.

Por lo tanto, el nivel de desarrollo exportador que exhiben las MYPES de la Región Tacna es bajo.

\section{PALABRAS CLAVE:}

Promoción de exportaciones, Desarrollo Exportador, Mypes exportadoras, Región Tacna.

\begin{abstract}
This is a basic, descriptive correlational research work because it tries to determine the relationship between variables studied, namely :Promotion of exports and exports development. We considered information obtained through a questionnaire titled " Promotion of exports and exports development in Mypes in Tacna ". It was applied to 99 Exporting Mypes in Tacna Region.

Our objective was to determine the influence of exports promotion in export development of MYPES in Tacna región in 2008-2009.

Our most important results were : Exporting MYPES in Tacna Region have a low level of knowledge on the benefits given by entities in charge of export promotion. Exporting MYPES in Tacna Region have a lower level of Access to programs and services for export promotion every year. Strategic alliances with institutions that promote exporting are mainly towards information and training about exporting, advising about exports was also given.
\end{abstract}

Also, the level of programs and services given by export institutions is very low.

Therefore, the level of export development achieved by MYPES in Tacna region is low.

\section{KEY WORDS:}

Exports promotion, export development, exporting MYPES, Tacna region. 


\section{INTRODUCCIÓN}

La promoción de exportaciones es ofrecida debido a la necesidad asumida para estimular las exportaciones, con el fin de mejorar la competitividad de las empresas en el mercado doméstico y en los mercados exteriores $\mathrm{y}$, disminuir el déficit de la balanza comercial.

Es preciso además indicar que las MYPES de la Región Tacna están dedicadas a la exportación de productos hidrobiológicos $(37,80 \%)$, aceituna $(32,10 \%)$, orégano $(18,40 \%)$, páprika $(6,20 \%)$, cochinilla $(1,10 \%)$, aceite de oliva $(1,30 \%)$, sandia $(0,80 \%)$, cebolla $(0,30 \%)$, otros $(1,80 \%)$; las que por su naturaleza y necesidad del exigente mundo competitivo, requieren realizar negocios con el exterior a fin de ampliar su mercado y alcanzar niveles de competitividad. Sin embargo, es de conocimiento que la mayoría de estas empresas desconocen los beneficios y servicios que brindan los organismos promotores de la exportación, restándoles de esta manera el nivel exportador a las MYPES de la Región Tacna, durante el período 2008 - 2009 .

Siendo la pregunta de investigación ¿En qué medida influyó la promoción de exportaciones, en el desarrollo exportador de las MYPES de la Región Tacna, en el periodo 2008 - 2009? Se plantea la siguiente hipótesis: La promoción de exportaciones influye en el nivel del desarrollo exportador de las MYPES de la Región Tacna, período 2008-2009.

La relevancia de la investigación está en su valor teórico al desarrollarse un nuevo instrumento para recolectar información y que puede ser útil para el sector empresarial, permitiendo conocer los beneficios y servicios que brindan los organismos promotores de la exportación.

\section{MATERIALES YMÉTODOS}

El presente estudio adoptó un diseño no experimental, de tipo descriptivo correlacional. Se considera no experimental; por cuanto no se manipula la variable independiente: promoción de exportaciones. Es de tipo descriptivo correlacional; porque se busca medir la relación de las variables (promoción de exportaciones y desarrollo exportador). La población de estudio está representada por la Mypes exportadoras de la Región Tacna.

Para efectos de estudio se determinó una muestra de 99 mypes exportadoras, aplicándose para la recolección de datos el cuestionario, que implementó la técnica de la encuesta. El instrumento de medición ha sido elaborado de acuerdo a las variables de estudio; y fue sometido a la prueba de confiabilidad y validez. Para su análisis e interpretación se adoptó un diseño de análisis estadístico descriptivo e inferencial.

Los resultados alcanzados fueron los siguientes:

\section{RESULTADOS}

INFLUENCIA DE LA PROMOCIÓN DE EXPORTACIONES EN EL DESARROLLO EXPORTADOR DE LAS MYPES DE LA REGIÓN TACNA.

\section{Gráfico $\mathrm{N}^{\circ} 01$}

Características de las Mypes: Tipo de producto que comercializan las MYPES de la Región Tacna

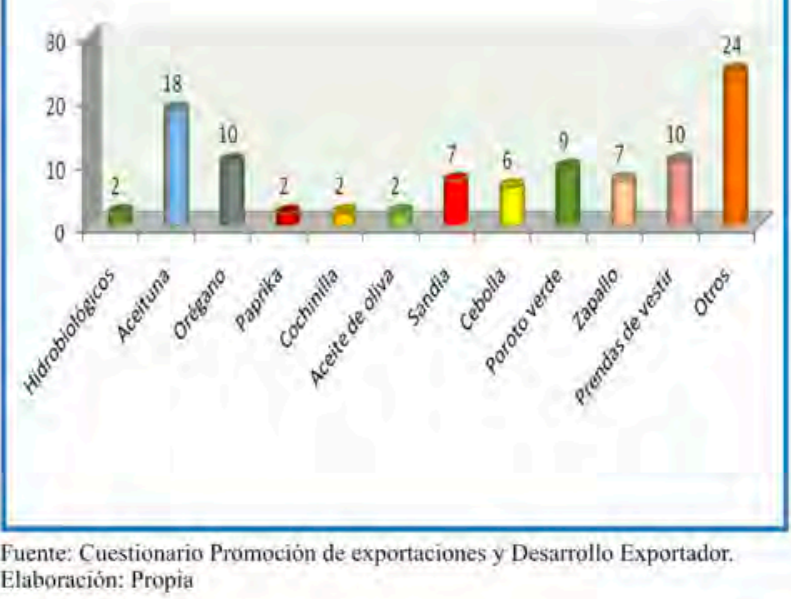

El $18 \%$ de las MYPES exportadoras de la Región Tacna comercializa aceitunas; el $10 \%$, orégano y prendas de vestir; el $9 \%$, poroto verde; el $7 \%$, sandía y zapallo; el $6 \%$, cebolla; el $2 \%$, cochinilla, paprika, aceite de oliva e hidrobiológicos; y, el $24 \%$, pertenece al rubro de otros (pisco, aceite de palta, madera tornillo, cochinilla seca, pulpa de fruta, choclo fresco, oxígeno, etc.).

La Región Tacna se caracteriza por comercializar productos del sector agrarario (cochinilla, cebolla, poroto verde, zapallo, otros); mientras que entre los de orden agroindustrial destacan aceituna, cebolla, orégano y ají páprika; los cuáles se comercializan sin valor agregado. 
Grálico $\mathrm{N}^{\circ} 02$

Características de las Mypes: Dimensión de las Mypes

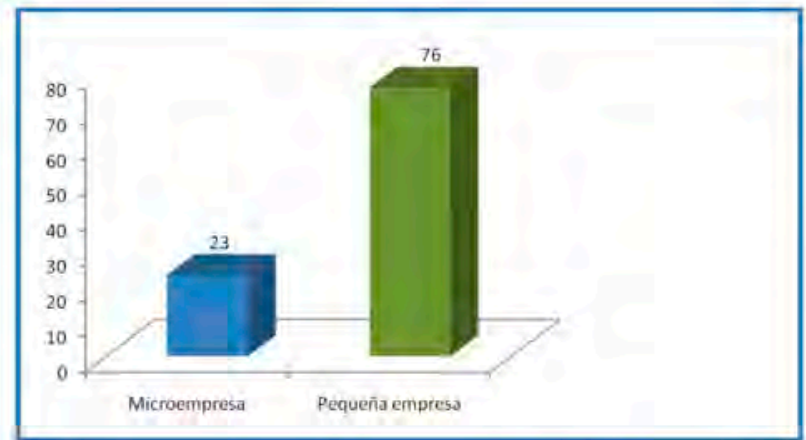

Fuente: Cuestionario Promoción de exportaciones y Desarrollo Expontadon Elaboración: Propia

El $76 \%$ los encuestados pertenece al estrato de la pequeña empresa; y, tienen entre 1 hasta 100 trabajadores. El 23\% pertenece al sector de la microempresa; $y$, tiene entre 1 hasta 10 trabajadores. Asimismo, el $71 \%$ de las pequeñas empresas registra ventas anuales desde 150 a 1700 Unidades Impositivas Tributarias; y, el $28 \%$ de las microempresas registra ventas anuales desde 1 a 150 Unidades Impositivas Tributarias.

El análisis anterior permite inferir que en la Región Tacna, la mayoría de las empresas que realizan sus operaciones de exportación, pertenece al estrato de la pequeña empresa.

\section{Gráfico $\mathrm{N}^{\circ} 03$}

Aspectos sobre promoción de exportaciones: Nivel de conocimiento que tiene las MYPES sobre los beneficios que brindan los organismos de promoción de exportaciones.

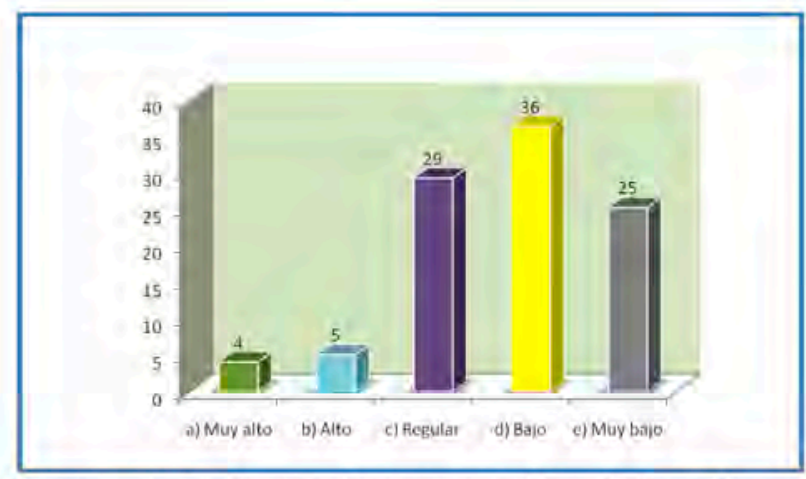

Fuente: Cuestionario Promocion de exportaciones y Desarrollo Exportador. Elaboración: Propia

El $36 \%$ de las MYPES encuestadas manifiesta que su nivel de conoeimiento en cuanto a los beneficios que brindan los organismos de promoción de exportaciones es bajo; y, sólo el 4\% declaró que su nivel de conocimiento era muy alto.

\section{Gráfico $\mathrm{N}^{\circ} 04$}

Aspectos sobre promoción de exportaciones: Organismos de promoción de exportaciones, de los cuales han hecho uso las MYPES de la Región Tacna.

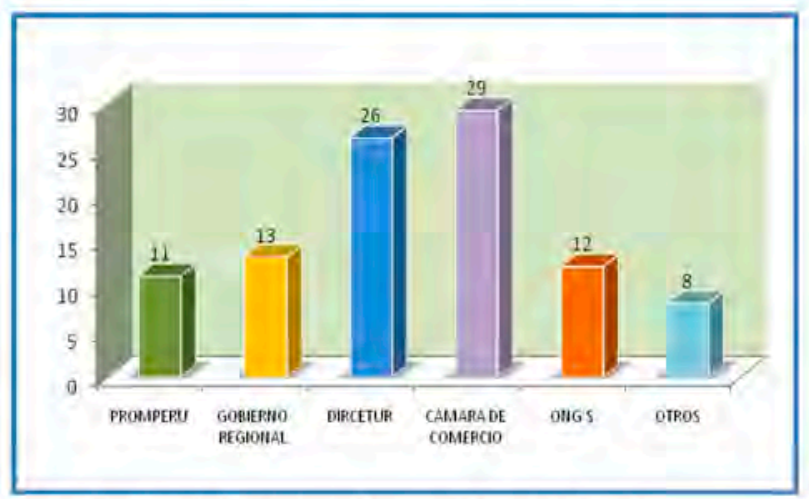

Fuente: Cuestionario Restricciones Internas. Elaboración: Propia

El 29\% de las MYPES exportadoras de la Región Tacna, ha hecho uso de los programas y servicios que brinda la Cámara de Comercio; el $26 \%$ señala que se ha beneficiado con los programas y servicios que ofrece DIRCETUR; $y$, el $8 \%$ manifiesta que han acudido a las ONG'S y a otros organismos.

\section{Gráfico $\mathrm{N}^{\circ} 05$}

Programas sobre promoción de exportaciones: Programa(s) para el mejoramiento de características organizacionales.

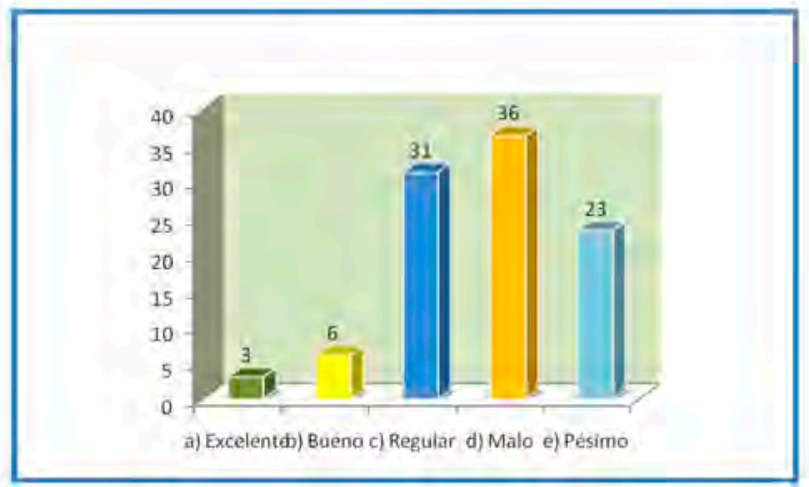

Fuente: Cuestionario Promoción de exportaciones y Desartollo Exportador. Elaboración: Propia

El $36 \%$ de los encuestados manifestó que el programa(s) para el mejoramiento de las características organizacionales es malo; el $31 \%$ señaló que es regular; el $23 \%$ indica que es pésimo; y sólo el $3 \%$, dice que es bueno y excelente. 


\section{Gráfico No 06}

Programas sobre promoción de exportaciones: Programa(s) de capacitación para el mejoramiento continuo de la calidad de los productos exportables.

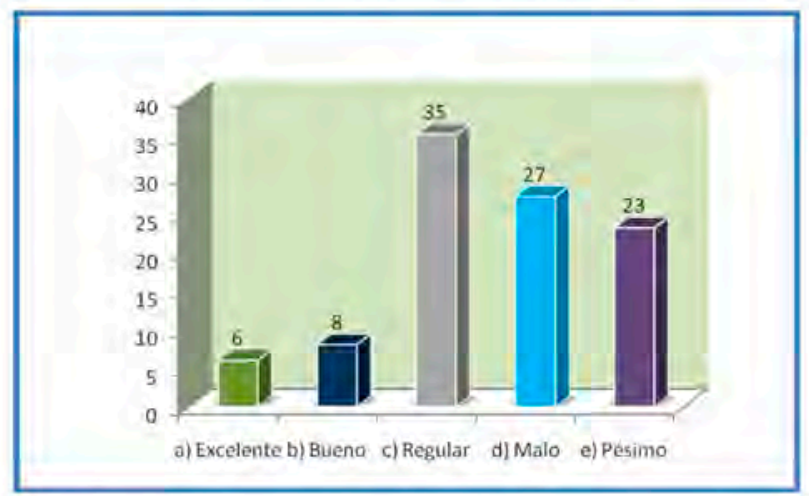

Fuente: Cuestionario Promoción de exportaciones y Desarrollo Exportador: Elaboración: Propia

E1 35\% de las MYPES exportadoras de la Región Tacna manifiesta que el programa(s) para el mejoramiento continuo de la calidad de los productos exportables es regular; el $27 \%$ manifiesta que es malo; y, el $23 \%$ señala que es pésimo.

\section{Gráfico $N^{\circ} 07$}

Servicios sobre promoción de exportaciones: Servicio brindado acerca de la información sobre mercados internacionales.

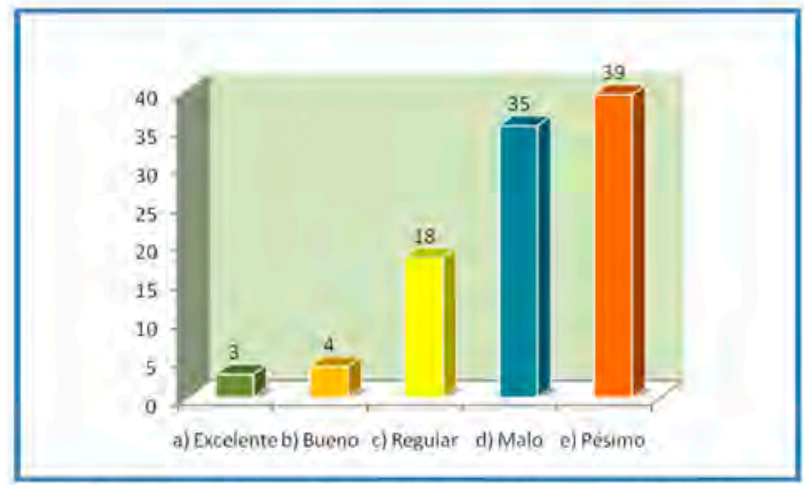

Fuente: Cuestionario Promoción de exportaciones y Desarrollo Exportador. Elaboración: Propia

E1 39\% de las MYPES exportadoras de la Región Tacna manifiesta que el servicio brindado acerca de la información sobre mercados internacionales es pésimo; el $35 \%$ señala que es malo; el $18 \%$ indica que es regular; el $4 \%$ dice que es bueno; y el $3 \%$ afirma que el servicio brindado acerca de la información sobre mercados internacionales es excelente.
Gráfico $N^{\circ} 08$

Servicios sobre promoción de exportaciones: Servicio brindado para el acceso a ferias internacionales.

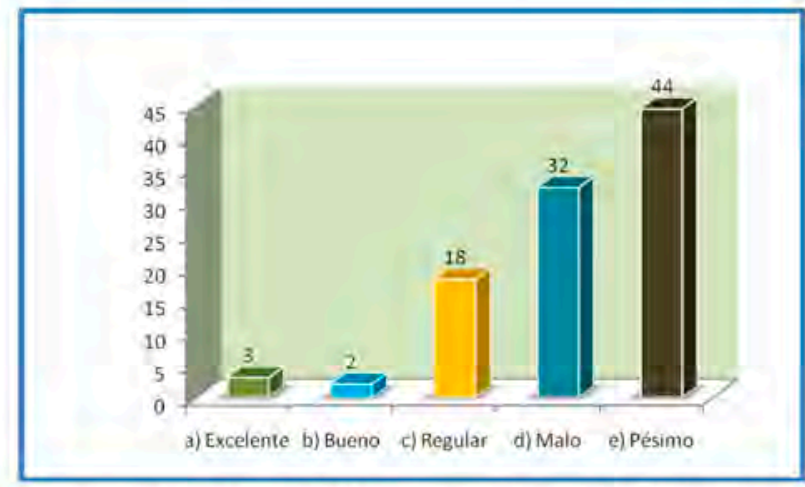

Fuente: Cuestionario Promoción de exportaciones y Desarrollo Exportador. Elaboración: Propia

El 47\% de las MYPES exportadoras de la Región Tacna manifiesta que el servicio brindado para el acceso a ferias internacionales es pésimo; el $29 \%$ señala que es malo; el $16 \%$ indica que es regular; el $3 \%$ dice que es excelente; $y$ el $4 \%$ afirma que el servicio brindado para el acceso a ferias internacionales es bueno.

\section{RESULTADOS \\ DESARROLLO EXPORTADOR DE LAS MYPES DE LAREGIÓN TACNA \\ Gráfico $\mathrm{N}^{\circ} 09$ \\ La organización define estrategias para alcanzar los objetivos previstos.}

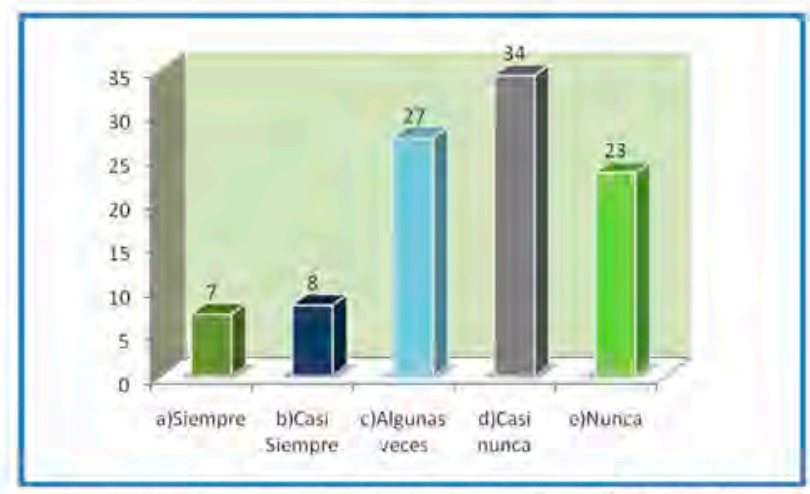

Fuente: Cuestionario Promoción de exportaciones y Desarrollo Exportador. Elaboración: Propìa

El 34\% de las MYPES encuestadas manifestó que casi nunca definen estrategias para alcanzar los objetivos previstos en su organización. 
Gráfico $N^{\circ} 10$

Las estrategias son definidas en atención a la planificación de la empresa.

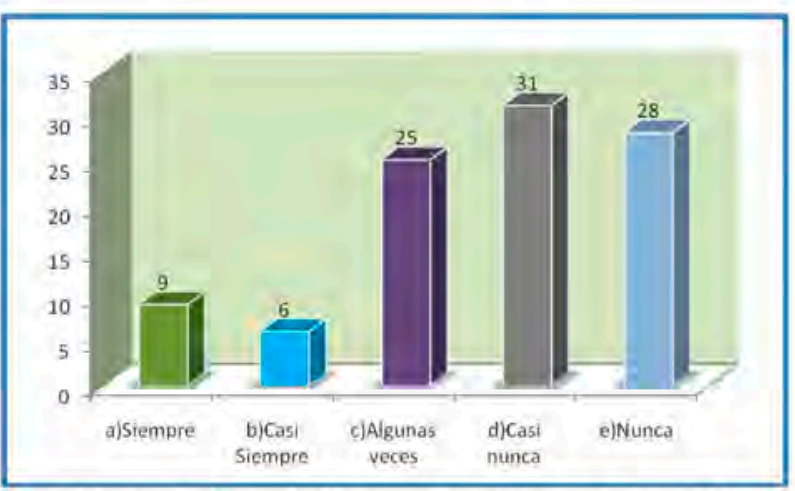

Fuente: Cuestionario Promoción de exportaciones y Desarrollo Exportador. Elaboraciỏn: Propia

El 31\% de las MYPES encuestadas manifestó que casi nunca las estrategias son definidas en atención a la planificación de la empresa.

\section{Gráfico $\mathrm{N}^{\circ} 11$}

Existe un plan de capacitación gerencial.

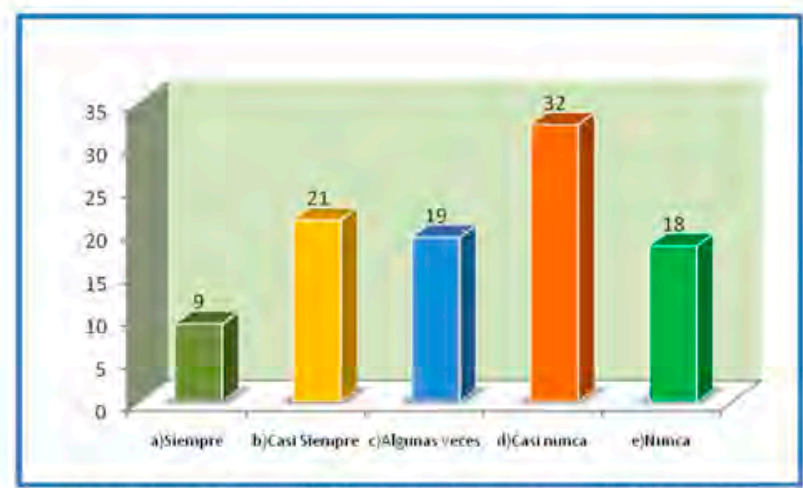

Fuente: Cuestionario Promoción de exportaciones y Desarrollo Exportador. Elaboración: Propia

El 32\% de las MYPES exportadoras de la Región Tacna manifestó que casi nunca existe un plan de cápacitación gerencial; el $21 \%$ señaló que casi siempre existía; el 19\% indicó que algunas veces había; el 18, 18\% de las MYPES exportadoras señaló que nunca lo hacía; y sólo el $9 \%$ afirmó que siempre existía en la organización un plan de capacitación gerencial.
Gráfico No 12

La organización mantiene alianzas con otras empresas del sector.

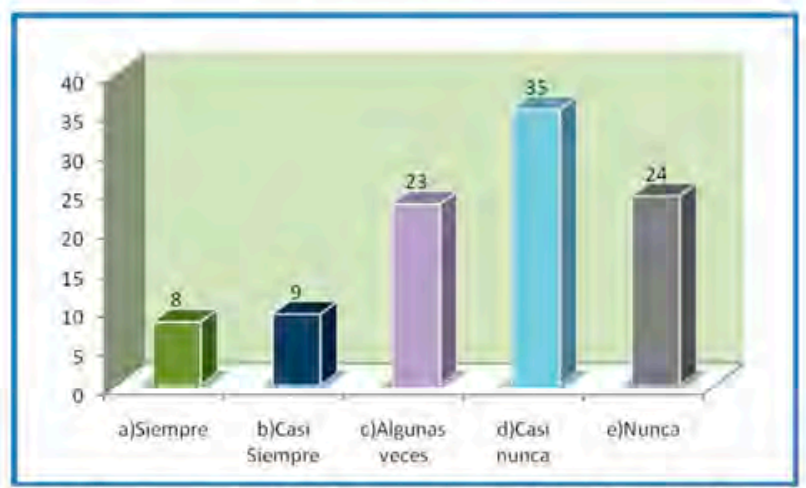

Fuente: Cuestionario Promoción de exportaciones y Desarrollo Exportador Elaboración: Propia

El 35\% de las MYPES exportadoras de la Región Tacna manifestó que la organización casi nunca mantiene alianzas con otras empresas del sector; el 24\% señaló que nunca lo ha hecho; y sólo el $8 \%$ indicó que siempre mantenia alianzas con otras empresas del sector.

\section{Gráfico $\mathrm{N}^{\circ} 13$}

Se han firmado contratos para la utilización de tecnología desarrollada por otras empresas.

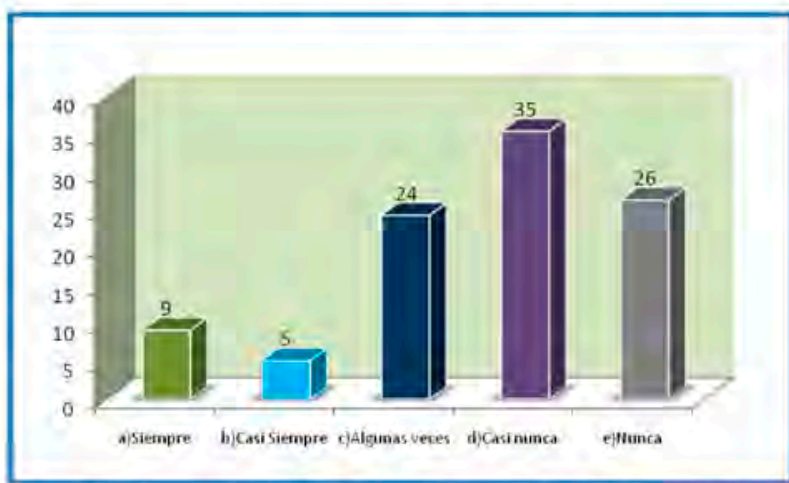

Fuente: Cuestionario Promoción de exportaciones y Desarrollo Exportador. Elaboración: Propia

El $45 \%$ de las MYPES exportadoras de la Región Tacna manifestó que casi nunca se han firmado contratos para la utilización de tecnología desarrollada por otras empresas; el $26 \%$ señaló que nunca se han firmado tales contratos; y sólo el $5 \%$ indicó que casi siempre se han firmado. 


\section{Gráfico N N 14 \\ Se utilizan tecnologías de información para la comercialización de los productos en los mercados internacionales.}

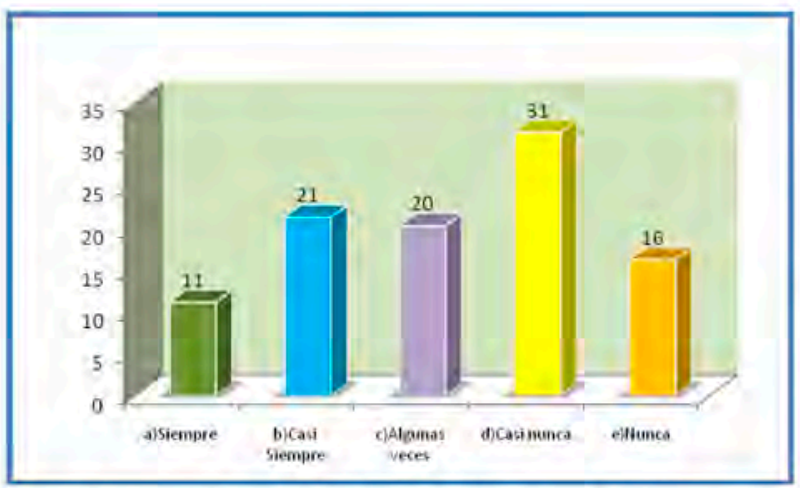

Fuente: Cuestionario Promoción de exportaciones y Desarrollo Exportador Elaboración: Propia

El 31\% de las MYPES exportadoras de la Región Tacna casi nunca utiliza tecnologias de información; y sólo el $11 \%$ manifestó que siempre las utiliza para la comercialización de los productos en los mercados internacionales.

\section{DISCUSIÓN}

La promoción de exportaciones se ha convertido en una politica prioritaria de los gobiernos de los países desarrollados, recién industrializados y, especialmente, en vias de desarrollo, al considerar las fuertes implicaciones que tiene la exportación en la política económica de una nación.

Según los resultados de la investigación se puede inferir que las MYPES exportadoras de la Región Tacna tienen un bajo conocimiento sobre los beneficios que brindan los organismos de promoción de exportaciones, puesto que el $36,36 \%$ manifestó que su nivel de conocimiento es bajo; y sólo el $4,04 \%$ indicó que su nivel de conocimiento es muy alto.

Asimismo, las MYPES exportadoras de la Región Tacna acceden a los programas y servicios de la promoción de exportaciones con menor frecuencia anualmente. Los objetivos de alianza con los organismos de promoción de exportaciones de las MYPES de la Región Tacna fueron en mayor medida hacia la información y capacitación sobre exportaciones; $y$, asesoría y exportaciones.

En cuanto el nivel de los programas y servicios brindado por los organismos de promoción de exportaciones se determinó que es bajo; ya que los programas sobre promoción de exportaciones tienen debilidades que deben superar.

Las MYPES de la Región Tacna descuidaron aspectos como la definición de estrategias para alcanzar los objetivos previstos, definir estrategias en atención a la planificación de la empresa, plan de capacitación gerencial, formación de alianzas con otras empresas del sector; lo que determina su nivel inferior en cuanto a su desarrollo exportador.

Por lo tanto, se concluye que la promoción de exportaciones influyó en el nivel del desarrollo exportador de las MYPES de la Región Tacna, período 2008-2009.

\section{REFERENCIAS}

1. BATEMAN, T., SNELL, S. (1999): Administración una ventaja competitiva. Cuarta Edición: Irwin $/ \mathrm{Mc}$ Graw Hill.

2. BRITO, Xiomara y otros (2001) Casos Simulados de Redes Empresariales entre Pequeñas y Medianas Empresas (PYMES) en Barquisimeto (Fase Teórica). Trabajo de Grado presentado para optar al Título de Licenciado en Contaduría Pública, Decanato de Administración y Contaduría (DAC) Universidad Centroccidental Lisandro Alvarado (UCLA).

3. CZINKOTA, M. (1996). Why national export promotion. International Trade Forum,

4. DIAMANTOPOULOS et al., 1993:5; Crick, 1995; Czinkota \& Wongtada 1997; Gillespie \& Riddle, 2004.

5. DIAMANTOPOULOS, A., Schlegelmilch, B. \& Tse, K. (1993). Understanding the role of export marketing assistance: empirical evidence and research needs. European Journal of Marketing, 27 (4), 5-18

6. Instituto Nacional de Estadística e Informática. Perú Compendio Estadístico 2005, Lima 2005

7. Instituto Nacional de Estadística e Informática. Compendio Cuentas Nacionales del Perú - Producto Bruto Interno por Departamentos 1994-2001. Lima, Febrero 2003.

8. Consejo Regional MYPE. Diagnóstico Regional Tacna de la Micro y Pequeña Empresa.

9. JONSON Gerry y Kevan Scholes. Dirección Estratégica. Edic. Pearson Educación, Madrid 2001.

10. GARCÍA Diaz, Jaime. Determinantes del Empleo Adecuado en las MYPE's en el Perú 1995-1998. Patrocinado por; BID, BM. Auspiciado por MECOVI PERÚ. 